\title{
Special issue on wireless multimedia sensor networks
}

With the availability of low-cost, small-scale imaging sensors, CMOS cameras, and microphones - all of which may ubiquitously capture multimedia content from the field - Wireless Multimedia Sensor Networks (WMSN) have been proposed and have received the immediate attention of the research community. WMSN applications, e.g., multimedia surveillance networks, target tracking, environmental monitoring, and traffic management systems, require effective harvesting and communication of event features in the form of multimedia such as audio, image, and video. To this end, additional challenges for energy-efficient multimedia processing and communication in WMSN, i.e., heterogeneous multimedia reliability definitions, tight QoS expectations, and high bandwidth demands, must be addressed as well.

For this very exciting and interdisciplinary topic, we have received 32 responses to our call-for-papers. As a result of a thorough review process by experts in the respective areas, this special issue consists of 6 selected papers describing the state-of-the-art, reliable and efficient multimedia processing and delivery solutions which are imperative for the realization of WMSN.

The first paper by N. Saxena, A. Roy, and J. Shin presents an adaptive contention, window-based quality of service (QoS) medium access control (MAC) protocol for wireless multimedia sensor networks. The proposed MAC protocol adopts the carrier-sense multiple access - collision avoidance (CSMA-CA) technique and adaptively adjusts the contention window depending on application-specific QoS-requirements for multimedia delivery over sensor networks as well as on the traffic and wireless channel characteristics. It also dynamically adapts its duty cycle based on the major application traffic pattern. Experimental results confirm the technique's operation and show that the technique preserves energy resources without compromising multimedia QoS requirements.

The second paper is authored by C. Na, Y. Yang, and A. Mishra and introduces an optimal guaranteed-time-slot (GTS) scheduling algorithm for time-sensitive multimedia delivery over sensor networks employing the low rate wireless personal area networks (LR-PAN) standard developed by the IEEE, i.e., IEEE 802.15.4. It presents a mathematical formulation to check the schedulability of a set of time-sensitive transactions with delay constraints in IEEE 802.15.4 networks operating in beacon mode. Based on this mathematical analysis, a novel GTS scheduling algorithm (GSA) - which is both work-conserving and optimal - is presented. Simulations reveal that GSA provides very high performance under bursty, periodic and aperiodic transmissions of transactions for multimedia delivery over low data-rate sensor networks.

The next paper, titled "Self-Orienting Wireless Multimedia Sensor Networks for Occlusion-Free Viewpoints" by $\mathrm{N}$. Tezcan and W. Wang, investigates the problem of self-orientation in WMSN in order to find the best possible orientation of multimedia sensors to maximize the multimedia coverage. To this end, a new distributed algorithm enabling sensors to calculate their directional coverage and self-configurable orientation is also proposed. It is verified through simulation experiments that the algorithm minimizes the negative effect of occlusions and overlapping regions in the field, and hence, the occlusion-free viewpoint approach significantly increases the multimedia coverage.

In their paper, "A Design and Implementation of Enhanced IEEE 802.15.4 for Supporting Multimedia Service in Wireless Sensor Networks", C. Suh, Z. Hameed, and Y.-B. Ko propose an enhancement to IEEE 802.15.4, named TEA-15.4, which adaptively adjusts the active period based on the traffic information. To detect data traffic in the network, the proposed scheme utilizes two techniques: Arbitrary Traffic Signal (ATS) and Traffic Time-Out (TTO). By utilizing these two techniques, the proposed TEA-15.4 can not only support enough data throughput to carry out multimedia communications, but also maintain lower energy consumption for multimedia sensors. They implemented their solution and the IEEE 802.15.4 full-standard on the TinyOS and, via experiments on a physical testbed, verified that TEA-15.4 is indeed a promising mechanism for WMSN.

Acoustic target tracking and classification is an important application of wireless multimedia sensor networks. The following paper, titled "Distributed Classification of Acoustic Targets in Wireless Audio-Sensor Networks" by B. Malhotra, I. Nikolaidis, and J. Harms, introduces a novel distributed framework to classify moving ground vehicles based on features extracted from the vehicles' acoustic 
signals. The framework enables the multimedia sensors to coordinate neighborhoods upon detection of a signal, partition the neighborhoods into active nodes and a master node, make measurements based on fast Fourier transform (FFT) and power spectral density (PSD) of the acoustic signals, and communicate this information to the master node in order to collaboratively classify the moving target with the $k$-Nearest Neighbor $(k-N N)$ classification method. The authors have conducted physical experiments using real acoustic signals from different vehicles recorded in their city and clearly shown the efficiency and effectiveness of their solution for acoustic target classification in WMSNs.

With the objective of high compression efficiency to reduce communication costs, many existing image compression techniques considered for sensor networks are extremely complex, such as JPEG2000. However, these algorithms mean complex hardware and a heavy processing load, and hence increased power consumption. In the last paper of this special issue, Q. Lu, W. Luo, J. Wang, and $\mathrm{B}$. Chen present a low-complexity and energy-efficient image compression scheme to reduce the hardware cost and the energy consumption in WMSNs. The compression algorithm in the proposed scheme greatly lowers the computational complexity and reduces the required memory, while it still achieves the required PSNR through the collaborative processing of tasks. It also dynamically updates transmission range to further reduce communication energy dissipation. The performance improvement in terms of energy consumption, image quality and WMSN lifetime is shown through extensive simulation experiments.

It has been a great pleasure to organize this special issue which reveals important research results in the broad field of multimedia sensor networks and highlights future research avenues on this emerging research field. We would like to thank Prof. Ian F. Akyildiz, Editor-in-Chief of Computer Networks (Elsevier) Journal, for initiating this special issue and for his support and encouragement. We would like to thank Dr. Harry Rudin, Editor-in-Chief (Special Issues) of Computer Networks (Elsevier) Journal, for giving us the opportunity to organize this special issue and for his editorial comments which have significantly improved the quality of this issue. We also thank all authors for their submissions and all reviewers for their diligent work in evaluating these submissions. We sincerely hope that you enjoy reading these distinguished papers and find appealing ideas for future explorative activities on this timely topic.

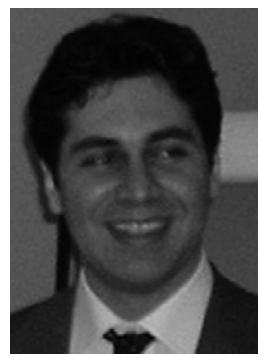

Ozgur B. Akan received the B.S. and M.S. degrees in electrical and electronics engineering from Bilkent University and Middle East Technical University, Ankara, Turkey, in 1999 and 2001, respectively. He received the Ph.D. degree in electrical and computer engineering from the Broadband and Wireless Networking Laboratory, School of Electrical and Computer Engineering, Georgia Institute of Technology, Atlanta, in 2004. He is currently an Associate Professor with the Department of Electrical and Electronics Engineering, Middle East Technical University. Dr. Akan is an Associate Editor for IEEE Transactions on Vehicular Technology, and Editor for ACM/ Springer Wireless Networks Journal. He is an IEEE Senior Member. His current research interests include wireless sensor networks, network information theory, signal processing for wireless networks, bio-inspired communications, and nano-scale communications.

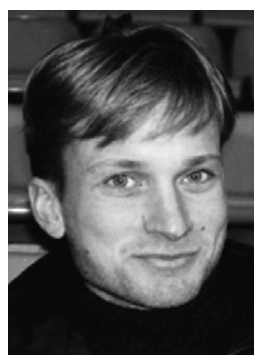

Pascal Frossard received the M.S. and Ph.D degrees, both in electrical engineering, from the Swiss Federal Institute of Technology (EPFL), Lausanne, Switzerland, in 1997 and 2000, respectively. Between 2001 and 2003 he was a member of the research staff at the IBM T. J. Watson Research Center, Yorktown Heights, NY, where he mainly worked on video coding and streaming technologies. Between 2003 and 2006, he was a Swiss National Science Foundation assistant professor at EPFL, where he now holds a tenure track position in the School of Engineering. His research interests include image representation and coding, nonlinear representations, visual information analysis, distributed information processing, joint source and channel coding, and multimedia communications. He is an Associate Editor of the IEEE Transactions On Circuits And Systems For Video Technology (2006-), of the IEEE Transactions On Multimedia (2004-). From 2004 to 2006, he has served as Vice-Chair of the IEEE Multimedia Communications Technical Committee.

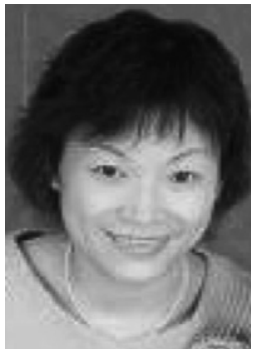

Qian Zhang received the B.S., M.S., and Ph.D. degrees from Wuhan University, China, in 1994, 1996, and 1999, respectively, all in computer science. She joined Hong Kong University of Science and Technology in September 2005 as an Associate Professor. She is now the associate director of the Digital Life Research Center of HKUST. Before that, she was at Microsoft Research, Asia, Beijing, China, starting in July 1999, where she was the research manager of the Wireless and Networking Group. Her current research interests are in the areas of wireless communications, IP networking, multimedia, P2P overlay, and wireless security. She is the Editorial Board Member of IEEE Transactions of Multimedia, IEEE Transactions on Wireless Communications, Elsevier Computer Communications, and Elsevier Computer Networks as well as the Associate Editor for IEEE Transactions on Vehicular Technologies. She is Vice-Chair and Award Committee Chair of the Multimedia Communication Technical Committee of the IEEE Communications Society. She is Chair of the Technical Affairs Committee of IEEE Asia Pacific Board (APB) of the IEEE Communication Society. She is also a member of the Visual Signal Processing and Communication Technical Committee and the Multimedia System and Application Technical Committee of the IEEE Circuits and Systems Society. She became a senior member of IEEE in 2004.

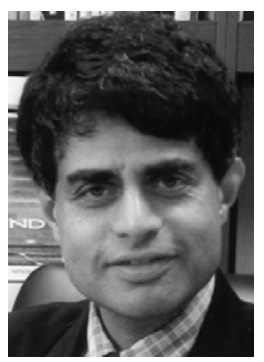

Nikil Jayant received the Ph.D. degree in electrical communication engineering from the Indian Institute of Science, Bangalore, India. As part of this doctoral program, he was a Research Associate at Stanford University, Stanford, CA, for one year prior to joining Bell Labs, Murray Hill, NJ, in 1968.

He joined the faculty of the Electrical and Computer Engineering Department, Georgia Institute of Technology (Georgia Tech), Atlanta, in July 1998, as a Georgia Research Alliance Eminent Scholar, as the John Pippin Chair in Wireless Systems, and as the Director of the Georgia Tech Wireless Institute. In April 1999, he created and became the first Director of the Georgia Tech Broadband Institute, with cross-campus responsibilities in research and industry partnership in broad-band access, lifestyle computing, and ubiquitous multimedia. In 
October 2000, he was named Executive Director of the Georgia Centers for Advanced Telecommunications Technology (GCATT). In 2004, he was named Director of Strategic Partnerships for Georgia Institute of Technology, with initial focus on industry partnerships in communications, computing and content. Earlier in his career at Bell Laboratories, he created and managed the Signal Processing Research Department, the Advanced Audio Technology Department and the Multimedia Communications Research Laboratory. He also initiated several new ventures for AT\&T and Lucent Technologies, including businesses in Internet Multimedia, Wireless Communications and Digital Audio Broadcasting. Recently, he served as the Chairperson of the National Academies Committee on Broadband Last Mile Technologies. He co-founded EGTechnology, an Atlanta-based startup company engaged in creating broad-band platforms, with initial focus on software for advanced television. He is also the Founder and President of MediaFlow, a consulting company. He has served on the Advisory Board of NTT-DoCoMo (USA), and is currently a Scientific Advisor to the Singapore Institute for Infocomm Research. His personal research has been in the field of digital coding and transmission of information signals. He has published 130 papers, authored or co-authored five books, and is also the author of 35 patents.

He has received several honors, including the IEEE Browder J.Thompson Memorial Prize Award (for the best IEEE publications by an author under thirty years of age, 1974), the IEEE Donald G. Fink Prize Paper Award (for the best tutorial in an IEEE publication, 1995), and the Lucent Patent Recognition Award (1997). In 1998, he was inducted into the New Jersey Inventors Hall of Fame. He was the Founding Editor-in-
Chief of the IEEE ASSP Magazine. He is a Fellow of the IEEE, a recipient of the IEEE Third Millennium Medal, and a member of the National Academy of Engineering.

Ozgur B. Akan

Middle East Technical University, Department of Electrical and Electronics Engineering, 06531 Ankara, Turkey

Tel.: +90 312210 2353; fax: +90 3122102304 E-mail address: akan@eee.metu.edu.tr

Pascal Frossard

Swiss Federal Institute of Technology (EPFL), Switzerland E-mail address: pascal.frossard@epfl.ch

Qian Zhang

Hong Kong University of Science and Technology, Hong Kong E-mail address: qianzh@cse.ust.hk

Nikil Jayant

Georgia Institute of Technology, United States E-mail address: jayant@ece.gatech.edu

Available online 25 June 2008 